\title{
Imanes de neodimio como propuesta de mínima intervención para procesos de conservación en soporte de tela pintados: corrección de deformaciones
}

\author{
Emanuel Sterp Moga, Alicia Sánchez Ortiz
}

\begin{abstract}
Resumen: En este artículo se propone una posible alternativa a los métodos tradicionales empleados en tratamientos de conservación-restauración para soportes de tela pintados siguiendo el criterio de mínima intervención. Se ha diseñado un bastidor auxiliar con sistema magnético (BASM) para la corrección de deformaciones y la sujeción perimetral de la obra con carácter temporal. A lo largo del trabajo se detalla la construcción del mecanismo propuesto y se analizan las pruebas experimentales que se han hecho sobre maquetas. Por último, se muestra la metodología seguida para su aplicación en un caso de estudio con obra real. Los resultados obtenidos con los sistemas magnéticos son muy prometedores, siendo una alternativa metodológica sencilla, de bajo coste, eficaz, reversible y respetuosa con el original.
\end{abstract}

Palabras clave: mínima intervención, pintura de caballete, conservación-restauración, imanes de neodimio, soportes de tela, digitalización 3D

\section{Neodymium magnets as a proposal for minimal intervention for conservation processes on painted fabric support: correction of deformations}

\begin{abstract}
This article proposes a possible alternative to the traditional methods used in conservation-restoration treatments for painted fabric supports following the criterion of minimal intervention. An Auxiliary Frame with Magnetic System (AFMS) has been designed for the correction of deformations and the perimeter fixation of the work on a temporary basis. Throughout the work, the construction of the proposed mechanism is detailed and the experimental tests carried out on models are analyzed. Finally, the methodology followed for its application in a case study with real work is shown. The results obtained with the magnetic systems are very promising, being a simple methodological alternative, low cost, effective, reversible and respectful with the original.
\end{abstract}

Keyword: minimal intervention, easel painting, conservation-restoration, neodymium magnets, canvas supports, 3D scanning

\section{Ímãs de neodímio como proposta de intervenção mínima para processos de conservação em suporte de telas pintadas: correção de deformações}

Resumo: Este artigo propõe uma alternativa possível aos métodos tradicionais usados em tratamentos de conservação-restauração para suportes de tecidos pintados, seguindo os critérios mínimos de intervenção. Uma estrutura auxiliar com sistema magnético (EASM) foi projetada para a correção de deformações e o perímetro periférico do trabalho em uma base temporária. Ao longo do trabalho, a construção do mecanismo proposto é detalhada e os testes experimentais que foram feitos nos modelos são analisados. Finalmente, a metodologia seguida para sua aplicação em um estudo de caso com trabalho real é mostrada. Os resultados obtidos com os sistemas magnéticos são muito promissores, sendo uma alternativa metodológica simples, de baixo custo, eficaz, reversível e respeitosa com o original.

Palavras-chave: intervenção mínima, pintura de cavalete, conservação-restauração, ímãs de neodimio, suportes de tela, digitalização 3D 


\section{Introducción}

La evolución de los criterios a aplicar en la conservaciónrestauración de la pintura de caballete elaborada sobre soporte textil, que tuvo su punto de inflexión en la Conferencia de Greenwich, celebrada en 1974, ha conllevado en los últimos años la revisión de las metodologías tradicionales y ha puesto en cuestionamiento algunos procedimientos considerados demasiado invasivos al comprometer la preservación del original en todos sus valores (Villers 2003; AA.VV. 2005; AA.VV. 2018). Ello ha contribuido a despertar el interés entre los profesionales del sector hacia el uso de nuevos materiales e instrumental específico que posibiliten realizar los tratamientos con una mayor precisión técnica, reduzcan los riesgos derivados de actuaciones invasivas, faciliten la acción localizada y se ajusten al concepto de mínima intervención, permitiendo futuras intervenciones (Ackroyd y Bomford 1991: 53-62; Charteris 1991: 141-145; Appelbaum 1987: 65-73; Smith 1988: 199-207).

Muchos de los problemas de conservación que se observan en la pintura de caballete sobre lienzo están causados por la inestabilidad estructural del soporte. Desde el mismo momento en que el pintor concluye su obra, la tela pintada y el bastidor que la soporta se ven sometidos a tensiones mecánicas como respuesta a las fluctuaciones en los parámetros de HR y Temperatura del ambiente expositivo (Hedley 1988: 133-148). Los textiles son materiales higroscópicos susceptibles de sufrir un proceso de envejecimiento con la consecuente pérdida de firmeza y elasticidad. Las fluctuaciones de humedad relativa provocan el hinchamiento y la contracción de las fibras que se ven afectadas, sobre todo, en su módulo de elasticidad longitudinal. Cuando la humedad relativa aumenta, la madera de los travesaños se hincha al mismo tiempo que las fibras del soporte textil se contraen. Estas reacciones, repetidas en el tiempo, pueden provocar una concentración de estrés mecánico que terminará dando como resultado una fatiga en la tela $y$, como consecuencia, el tejido experimentará un alargamiento o estrechamiento de las fibras que lo compone, con la aparición de deformaciones, de mayor o menor extensión, debido a la concentración del estrés en las esquinas por la presencia de los listones del bastidor (Mecklenburg 1982), roturas en los hilos, y si no se toman medidas adecuadas de conservación, con una repercusión directa sobre los estratos pictóricos (Sánchez Ortiz 2012: 94-95).

Para resolver los problemas descritos, todos ellos ligados al comportamiento mecánico de los soportes pictóricos, Roberto Carità, teórico del arte y técnico del Instituto Centrale per il Restauro (ICR) de Roma, llevó a cabo, en 1950, los primeros estudios científicos con el objetivo de demostrar, desde un punto de vista físico, la cuantificación de las fuerzas de tensión mecánica. Propuso un innovador prototipo de bastidor con muelles para regular la distribución de las mismas en el conjunto de la obra y lo aplicó a algunos cuadros, como la Degollación de San Juan Bautista y el San Jerónimo (Carità 1955: 165170); en ambos casos, el lienzo quedaba suspendido dentro de un perímetro elástico y se lograba mantener una distribución uniforme de las tensiones con ayuda de un sistema de muelles anclados en el reverso del bastidor, con lo que se evitaban las citadas concentraciones de estrés en las esquinas. Años después, Rigamonti diseñó un nuevo mecanismo caracterizado por la incorporación de aluminio y dotado de un sistema de tensión compuesto por varilla roscada que posibilitaba la apertura gradual de los ángulos del bastidor (Fabeiro, Hamada, Illán y Romero 2005). Cabe destacar también la investigación liderada por Antonio laccarino quien, en colaboración con Carlo Serino, ha elaborado un nuevo modelo de bastidor con sistema de tensión constante mediante muelles que permite a la tela permanecer flotando sobre los travesaños originales (Gironés, laccarino y Serino 2010: 209-210). Similar en cuanto a su comportamiento mecánico es el funcionamiento del bastidor TWP Stretcher, que tiene como particularidad la incorporación de un sistema de railes en los cantos interiores de los listones y de tornillos de mariposa en los ángulos (Tsang, Madruga, Williams, Pelasara y Patterson 2013: 92-94).

La innovación tecnológica en el campo de la conservaciónrestauración de bienes culturales ha propiciadoel desarrollo de utensilios y equipos de apoyo que permiten corregir las deformaciones y tratar los daños que comprometen la estabilidad estructural del soporte pintado. En este sentido, los imanes de neodimio -utilizados por primera vez a finales de 1980 para la exposición de textiles extremadamente delicados o que mostraban un estado especialmente comprometido-, suponen una alternativa de gran interés al servir de sustitutos a los métodos de instalación tradicional con los cuales existe una alta probabilidad de causar algún tipo de daño sobre el original (Derbyshire 2005: 2-4). La utilidad de estos sistemas imantados de montaje ha ido ampliándose y hoy en día encuentran aplicación en diversas tipologías de objetos y obras de arte, tanto en el montaje de exposiciones temporales como en el almacenaje, sobre todo de textiles y de documentos gráficos (Noehles y Niehus 2014: 236). En cuanto al empleo de sistemas imantados en procesos de conservación-restauración destaca el kit magnético puesto a punto por los italianos Luigi Rella y Lucia Sanccani, que consta de dos placas de acero galvanizado y de varios imanes, los cuales se sitúan sobre las zonas a tratar del lienzo sin necesidad de proceder al desmontaje de su bastidor (Rella y Saccani 2006: 17-19; Bestetti 2005: 336-343). Tratamientos de corrección de deformaciones con imanes se han llevado a cabo tanto en pintura antigua (Wroczynski 2013) como contemporánea (Art Galery New South Wales 2015), si bien hasta el presente no han encontrado una amplia difusión entre los restauradores.

El presente estudio muestra los resultados obtenidos durante el Trabajo Fin de Máster en Conservación del Patrimonio Cultural, realizado en la Facultad de Bellas Artes de la Universidad Complutense de Madrid (Sterp, 2018). 


\section{Objetivos}

El objetivo principal ha sido demostrar la utilidad de los sistemas magnéticos como alternativa válida en procesos de conservación-restauración centrados en la corrección de deformaciones del soporte textil en pintura, haciendo especial hincapié en el criterio de mínima intervención y en la retratabilidad de las operaciones.

Para lograr este objetivo principal se han establecido cinco objetivos específicos:

1. Conocer la fuerza de adhesión que debe tener un adhesivo para soportar las fuerzas de tracción a las que estará sometido el imán de neodimio colocado en los listones del bastidor auxiliar.

2. Obtener datos relativos a la fuerza de tracción que soportan los sistemas magnéticos seleccionados sobre distintas telas, naturales y sintéticas, sin preparación y con estratos pictóricos.

3. Diseñar diferentes prototipos de bastidores auxiliares y ensayar posibles soluciones de tratamientos sobre modelos virtuales con ayuda de la fotogrametría.

4. Realizar maquetas físicas que reproduzcan situaciones habituales de deterioro en soportes de tela pintados, con el fin de proceder a las intervenciones seleccionadas.

5. Implementar en obra real los resultados alcanzados con las maquetas físicas y el estudio virtual 3D.

\section{Parte experimental}

Se ha elegido como caso de estudio la obra Virgen con el Niño, una pintura al óleo sobre lienzo, con unas dimensiones de $104 \times 76 \mathrm{~cm}$, de autor anónimo, realizada en el siglo XVIII y perteneciente a la Diócesis de Salamanca. Entre las patologías más relevantes que presentaba antes de su intervención cabe destacar el estado de debilitamiento de las fibras del soporte textil como consecuencia de procesos de oxidación provocados por las condiciones adversas a las que ha estado expuesto el cuadro.
Previo al estudio del nuevo Bastidor Auxiliar con Sistema Magnético (BASM), se ha procedido a digitalizar el lienzo en 3D mediante la técnica de la fotogrametría por medio del software Agisoft PhotoScan ${ }^{\circledast}$ Standard Edition. Posteriormente se han diseñado diferentes prototipo del BASM en 3D mediante el software Blender ${ }^{\circledR}$, procediendo al estudio del funcionamiento y utilización del mismo.

Haciendo referencia a los diferentes tipos de tejidos e hilos para comprobar la tensión que son capaces de soportar, se han realizado ensayos de tracción mediante la preparación de una serie de maquetas con un dinamómetro Colometer IF67 y por medio de tensión meter HT-6510N, en los casos necesarios para saber la tensión de la superficie en $\mathrm{N} / \mathrm{cm}^{2}$. Asimismo, se ha estudiado la fuerza de presión que estos sistemas magnéticos ejercen sobre la superficie pictórica.

A partir de todos estos estudios y diseños previos, se ha ensayado primero con un modelo físico para la intervención sobre maquetas de diferentes supuestos específicos de problemáticas habituales en el soporte de tela pintados. En base a los resultados obtenidos, se ha desarrollado el sistema adaptado a la problemática de la obra real.

\section{Estudio de la obra digitalizada y elaboración de diferentes diseños en 3D}

Como primer paso, se ha procedido a digitalizar el cuadro seleccionado con la técnica de la fotogrametría. Para ello se ha utilizado el software Agisoft PhotoScan ${ }^{\circledast}$ Standard Edition [figura 1]. El levantamiento fotográfico se ha hecho con una cámara Canon $80 \mathrm{D}$, dotada de un objetivo de $18-135 \mathrm{~mm}$. Las capturas fotográficas se han realizado girando alrededor de la obra con la cámara y se han utilizado los siguientes parámetros de captura: ISO 100, diafragma de f/11 y velocidad de obturación de 1/800. Las 172 fotografías obtenidas durante el proceso se han capturado en formato RAW para poder revelarlas digitalmente por medio de Adobe $^{\circledR}$ CameraRaw, en

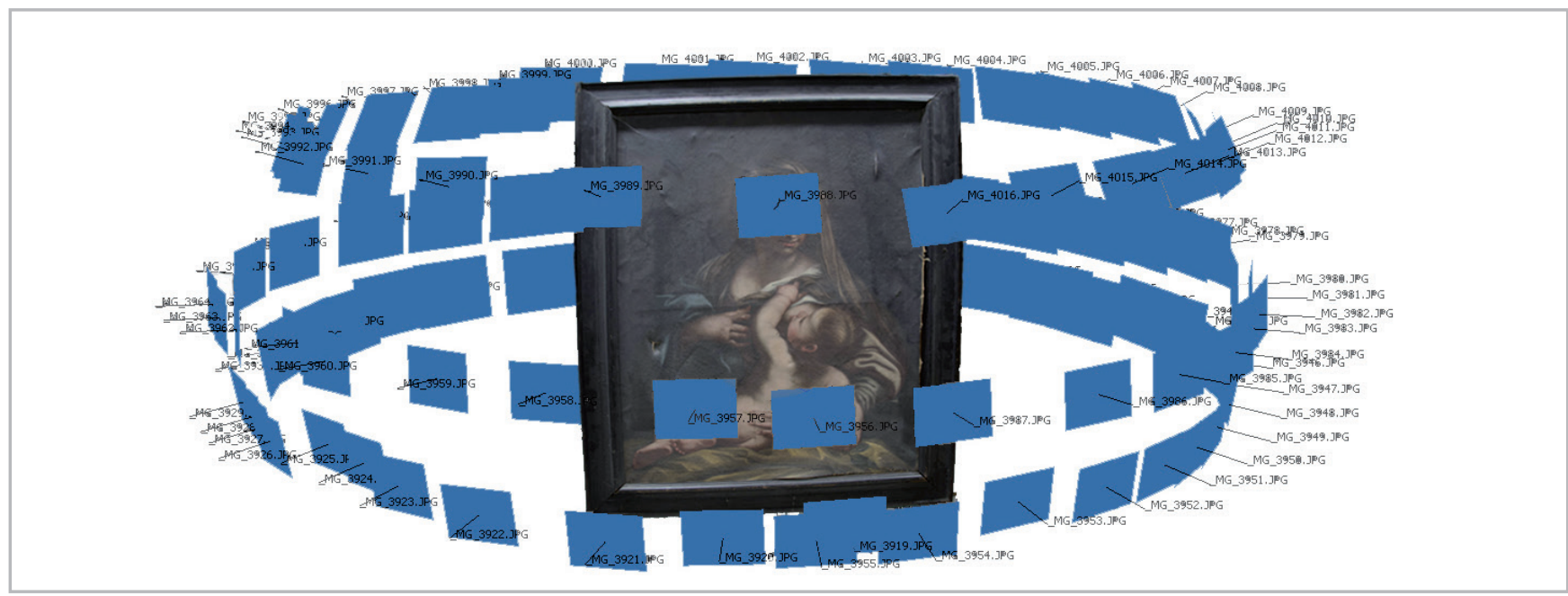

Figura 1.- Realización de la fotogrametría. Obra: Virgen con Niño, anónimo, escuela española, siglo XVIII. @Emanuel Sterp Moga 
Photoshop CC. Con la finalidad de minimizar el efecto provocado por los brillos se ha empleado un filtro polarizador de la marca Hoya PL-CIR $67 \mathrm{~mm}$. El conjunto de fotografías se ha ensamblado con el software Agisoft PhotoScan ${ }^{\circledast}$ Standard Edition. Debido al escaso grosor del lienzo ha sido indispensable trabajar con dos baterías de imágenes, lo que ha permitido obtener la imagen del 3D tanto del anverso como del reverso por separado, y, a continuación, proceder a soldarlas entre sí.
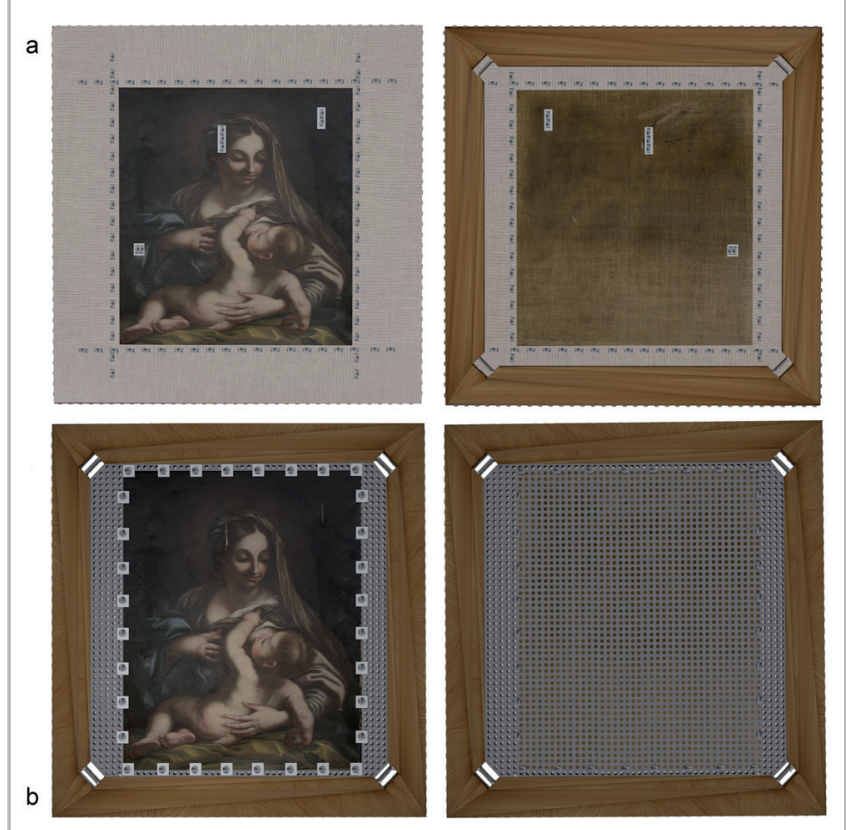

Figura 2.- Diseños de las diferentes intervenciones (a) Corrección de deformaciones (b) Sujeción perimetral de la obra. OEmanuel Sterp Moga

Los diferentes prototipos virtuales del nuevo bastidor con sistema magnético (BASM) se han diseñado en 3D con ayuda del software Blender ${ }^{\circledR}$ [figura 2]. El diseño digital de este se ha realizado a través de un modelado poligonal, donde cada objeto creado está compuesto por vértices, aristas y polígonos que van conectados entre sí y permiten de una manera muy flexible el modelado de todas las piezas. El estudio virtual mediante el modelo fotogramétrico obtenido y los diseños de los bastidores realizados han sido claves para profundizar en la correcta construcción y ajustar el funcionamiento del BASM con el fin de abordar los procesos de corrección de deformaciones y sujeción temporal de la obra estudiada.

\section{Selección de los imanes}

Se han seleccionado imanes de neodimio con magnetización axial, en forma de discos, cilindros y bloques de diferentes tamaños. La aleación (NdFeB), con la que están fabricados, los convierte en el tipo de imanes permanentes de tierras raras con mayor fuerza y densidad en el campo magnético (Spicer 2010: 3). Su revestimiento
(NiCuNi) los protege frente a los procesos de corrosión y aumenta su resistencia. Cada tipo de imán es clasificado mediante unas letras y un número determinado (N42, N45 o 45SH). Los números representan la magnetización, es decir, la calidad del material magnético empleado en el volumen del interior de cada imán. Cuanto mayor sea el número indicado más frágil será el imán y mayor riesgo de rotura soportará (Spicer 2017: 60-61). Las letras indican la temperatura máxima a la que se puede utilizar el imán. En base a estas características se han seleccionado los siguientes imanes: S-15-03-R, S-12-3-N, S-10-2-N, S-8-2-N, S-5-8-N, S-5-3-N, Q-8-8-4-N, Q-10-10-3-N, Q-25-6-2-SN y Q-20-10-2N.

En cuanto al montaje del mecanismo, cualquier sistema magnético permite tres combinaciones posibles: un conjunto compuesto por imán-imán, por imán-metal ferromagnético, o por metal ferromagnético-imánmetal ferromagnético (Spicer 2017: 64-65). En todas ellas será necesario disponer de una plancha metálica para conseguir crear el magnetismo deseado. Es importante destacar el factor de reversibilidad en el montaje del mecanismo independientemente del tipo de imán que se elija; en la práctica, el montaje de este se puede invertir rápidamente, de modo que se evita tener que recurrir al empleo de otros sistemas más invasivos y dañinos. Para favorecer la manipulación de los imanes se recomienda emplear una hoja de Mylar ${ }^{\circledast}$ como interface entre el imán y el objeto (Spicer 2010: 3). Por último, es importante conocer la máxima fuerza de sujeción que se producirá entre los dos imanes que actúan como pinza para sujetar la obra. Cuando se produce una mínima separación, la intensidad se reduce inversamente proporcional al cuadrado de la distancia que hay entre los polos (Tipler 1992: 612). Conviene tener en cuenta que el espesor de la obra influirá de manera directa en la fuerza de los imanes.

\section{1a Fase. Ensayos de tracción y presión sobre estratos pictóricos}

-Ensayo 1. Para determinar la fuerza máxima que es capaz de soportar el adhesivo a emplear en la colocación de los imanes distribuidos en los cantos exteriores el bastidor, se han llevado a cabo ensayos con un dinamómetro Colometer IF67. Sobre la maqueta $A$ se han testado los imanes del tipo S-12-3-N (N45), S-8-2-N (N45), S-10-2-N (N42), S-5-8-N (N45), S-5-3-N (N42), que previamente se habían adherido a una muestra de madera con dos adhesivos diferentes, Pattex ${ }^{\circledast}$ Nural 27 y Araldite ${ }^{\circledR}$ Standard extra fuerte [figura 3 a]. La elección de estos adhesivos es debido a que se adhieren tanto a materiales porosos como no porosos. Como características específicas la primera resina tiene una fuerza de tracción de $100 \mathrm{~kg} / \mathrm{cm}^{2}$, una temperatura de transición vítrea $(\mathrm{Tg})$ de $25^{\circ} \mathrm{C}$ y un tiempo de manipulación de 15 minutos; en cuanto a la segunda, ofrece una resistencia a la tracción de $350 \mathrm{~kg} / \mathrm{cm}^{2}$, una temperatura de transición vítrea $(\mathrm{Tg})$ de $40^{\circ} \mathrm{C}$ y un tiempo de trabajo mucho más amplio, hasta los 90 minutos. 
-Ensayo 2. Se han seleccionado tres tejidos sintéticos, LIPARI $\mathrm{g} / \mathrm{m}^{2} 260$, ISPRA $\mathrm{g} / \mathrm{m}^{2} 130$ y ISPRA $\mathrm{g} / \mathrm{m}^{2} 100$, y uno natural, Lino $2297 \mathrm{~g} / \mathrm{m}^{2}$ 170, y se ha procedido a medir la fuerza de tracción que son capaces de soportar sometidos a una determinada tensión constante durante 48 horas. El tejido de lino se ha elegido dado que es el soporte textil más empleado en la pintura de caballete tradicional, además, es bastante fuerte y presenta una gran resistencia a las deformaciones y roturas. Se ha considerado oportuno también testar telas de poliéster dada a su considerable estabilidad dimensional y su elevada resistencia a la tracción y humedad, imprescindible para el proceso de corrección de deformaciones. Asimismo, se han seleccionado diferentes gramajes para observar el comportamiento y resistencia durante la tensión aplicada con los imanes de neodimio. Los resultados obtenidos muestran que las telas sintéticas proporcionan una mejor estabilidad durante el proceso de tensado $\mathrm{y}$, entre ellas, la tela sintética LIPARI, con un gramaje $\left(\mathrm{g} / \mathrm{m}^{2}\right) 260$, es la que mayor tensión aguanta en relación a los sistemas magnéticos testados.

Con el presente ensayo se buscan alcanzar dos resultados. El primero consiste en analizar si las telas sometidas a una tensión constante durante 48 horas mediante el sistema magnético seleccionado, se deforman o pierden tensión, y el segundo, en examinar hasta que fuerza de tracción las telas son capaces de resistir mediante los imanes de neodimio. Para ello, se ha elaborado una maqueta T compuesta por un bastidor e imanes. Cada una de las muestras ha sido con mediante los imanes de neodimio,
S-12-3-N, S-8-2-N, S-10-2-N, S-5-8-N, S-5-3-N, adheridos por un lado. Para tensar los tejidos, estos se han sujetado mediante los imanes seleccionados adheridos a uno de los listones con los adhesivos Pattex ${ }^{\circledR}$ Nural 27 y Araldite $^{\circledR}$ Standard extra fuerte, y el extremo opuesto del tejido se ha sujetado con los imanes pertinentes adheridos a una estructura metálica enganchada a un dinamómetro. De esta manera, al tensar los tejidos se puede medir la fuerza de tracción realizada [figura 3 b]. Se ha elegido este método de ensayo con el objetivo de que cada restaurador tenga al alcance unos medios asequibles para realizar él mismo las pruebas, adaptándolo a la singularidad de la obra si fuera necesario. El experimento ha consistido en comprobar la fuerza de tracción sobre las diferentes telas elegidas, primero sólo con la colocación de un imán, luego con dos $y$, por último, con tres imanes, de manera que se pudiesen determinar la resistencia a la tracción de los tejidos elegidos y la capacidad de sujeción de los imanes.

-Ensayo 3. Dada la alta atracción que se produce en el campo magnético entre dos imanes, es posible que existan riesgos de provocar, por presión excesiva, algún tipo de aplastamiento en la superficie pictórica. Por ello, se ha considerado conveniente a la hora de seleccionar el tipo de imán tener en cuenta el tamaño y la forma del imán, el número de imanes, la fuerza de tracción, la fuerza constante, la técnica pictórica y la estructura estratigráfica de la obra. De igual modo, es esencial prever algunas medidas preventivas como proteger estos sistemas frente a los choques mecánicos, al calor, a la humedad y evitar que se produzca un campo desmagnetizado (Spicer, 2017: 68).
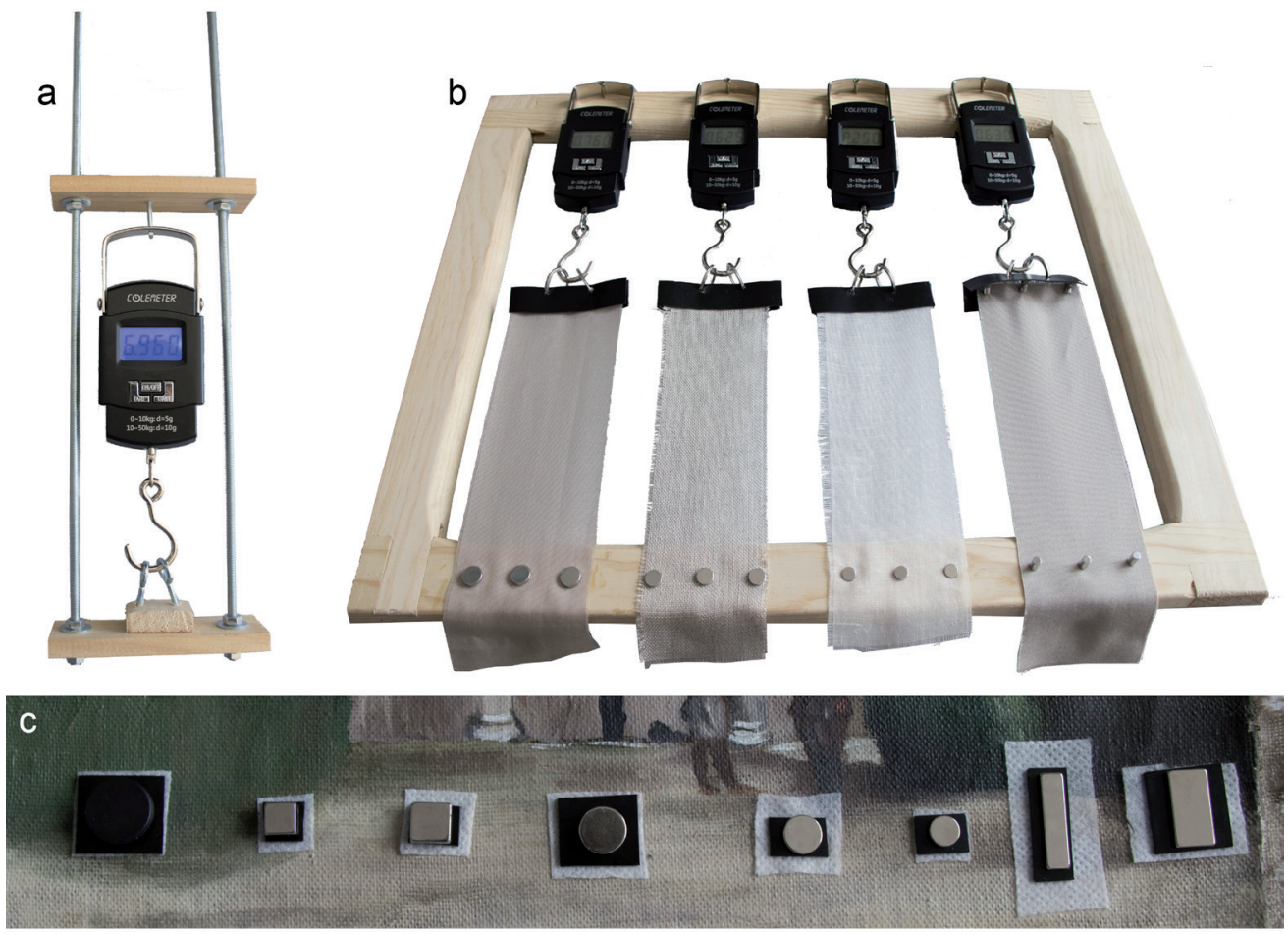

Figura 3.- a) Pruebas de tracción de los adhesivos mediante la colocación de los imanes de neodimio. b) Pruebas de tracción con las diferentes muestras de telas con imanes. c) Aplicación de imanes sobre la capa pictórica al óleo. @ Emanuel Sterp Moga 
La maqueta $\mathrm{P}$ está compuesta por un lienzo de lino con preparación tradicional de cola de conejo y yeso extra de Bolonia y ha sido pintada al óleo. Durante 48 horas las muestras se han sometido a una presión constante con los imanes S-15-03-R, S-12-3-N, S-10-2-N, S-5-3-N, Q-8-84-N, Q-10-10-3-N, Q-25-6-2-SN, Q-20-10-2N. Se ha evitado que estos entrasen en contacto directo con la superficie pintada mediante la interposición de una lámina de Tyvek $^{\circledast}$ y de una cinta de neopreno de $1 \mathrm{~mm}$ de grosor, colocada encima de ella [figura $3 \mathrm{c}$ ].

\section{2a Fase. Fabricación del prototipo de Bastidor Auxiliar con Sistema Magnético (BASM)}

Para la construcción del BASM se ha elegido como especie leñosa el abeto laminado, debido a que presenta unas propiedades físicas consideradas apropiadas, como una densidad aparente del $12 \%$ de humedad y $0,45 \mathrm{~kg} / \mathrm{m}^{3}$; tiene, además, un coeficiente de contratación volumétrico de $0,44 \%$ y una resistencia a la flexión estática de $710 \mathrm{~kg} /$ $\mathrm{cm}^{2}$, lo que la convierte en una madera muy estable. El bastidor tiene unas dimensiones de 54,5 × 45,5 × 1,7 cm [figura 4]. Los cuatro listones se encuentran cortados, en sus extremos, en ángulo de $45^{\circ}$ y constan de un mecanismo de tensión compuesto por espigas de acero inoxidable, así como varillas con rosca y tuercas.

El primer mecanismo presenta un sistema telescópico donde un tubo permanente en uno de los dos listones penetra en el listón contiguo dentro de otro cilindro metálico, con un diámetro de $8 \mathrm{~mm}$ y $4 \mathrm{~cm}$ de profundidad. De esta manera, la espiga entra y sale del orificio según se abra o cierre el mecanismo mediante el sistema de varilla roscada y tuercas. Se consigue así disminuir notablemente la fricción entre la madera y el acero; además, cuando se realice la apertura del bastidor, la espiga de acero inoxidable reforzará las esquinas evitando que se muevan durante la operación.

Respecto al segundo mecanismo -compuesto por varillas con rosca y tuercas-, estas van colocadas en la parte interior del listón y se encuentran alojadas dentro de un cilindro metálico que presenta un diámetro de $10 \mathrm{~mm}$ y 6 $\mathrm{cm}$ de profundidad. A diferencia del sistema telescópico anterior, las varillas no se hallan fijadas en ninguno de los listones, con la intención de favorecer el proceso de apertura en los dos lados. Para mejorar el acceso a las tuercas y la manipulación de las mismas con una llave, los listones en los cantos interiores presentan un corte a modo de caja. Cabe destacar que los dos sistemas están colocados a la misma altura del listón. Es un mecanismo sencillo, rápido, preciso y muy resistente.

En el canto externo de cada listón se han taladrado una serie de orificios, de $0,3 \mathrm{~cm}$ de profundidad y 5 milímetros de diámetro, separados uno de otro a una distancia de 1,5 $\mathrm{cm}$. En ellos se han alojado imanes de neodimio de esas mismas dimensiones y se han adherido mediante resina epoxídica Araldite ${ }^{\circledR}$ Standard extra fuerte, de forma que todos ellos quedan a ras de la superficie del listón. Dichos imanes sujetaran diferentes bandas textiles que ayudaran a concluir el proceso de corrección de deformaciones.

Por último y respecto a los cantos inferiores de cada listón, se ha realizado en su línea media un surco longitudinal, de $1 \mathrm{~cm}$ de profundidad y $0,2 \mathrm{~cm}$ de ancho, en el que se puede alojar una plancha de acero inoxidable cuya función será actuar a modo de base para soportar el lienzo que se va a intervenir. Cabe destacar que esta plancha solamente se utiliza para este fin y durante el proceso de corrección de deformaciones no es necesaria.

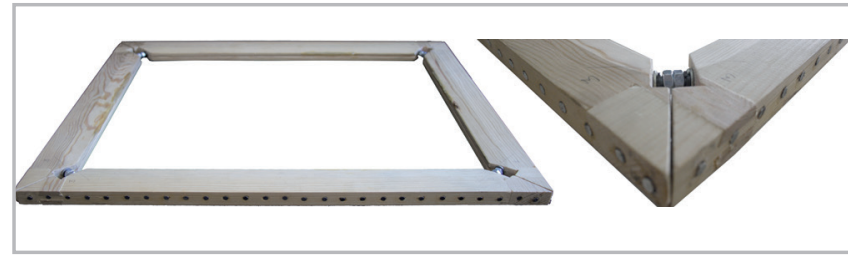

Figura 4.- Imagen general y de detalle del Bastidor Auxiliar con Sistema Magnético (BASM). @Emanuel Sterp Moga

\section{$3^{a}$ Fase. Preparación de facsímiles}

Durante el proceso de tensado -llevado a cabo sobre las maquetas-, se ha observado que los desgarros, las lagunas y los rotos practicados en ellas experimentan ligeras deformaciones, de entre $0,5-1 \mathrm{~cm}$, y que las zonas con pérdidas de soporte textil liberan tensiones mecánicas con la formación de pliegues. Para solventar estos inconvenientes se consideró apropiado colocar en las áreas afectadas por este tipo de daños diferentes imanes Q-20-10-02- N, que se dispusieron tanto en la tela como sobre los estratos pictóricos, protegidos en este último caso con una tira de Tyvek $^{\circledR}$ y una cinta de neopreno. Se ha seleccionado este tipo de imán debido a su forma y a sus dimensiones, ya que abarca más superficie en el soporte textil en relación a lo que ocurre con otro tipo de imanes, teniendo todos ellos unas características físicas similares. Es importante señalar que este sistema ha de estar perfectamente montado, de tal manera que el corte del desgarro quede centrado en la base del imán.

-Prueba A. Para testar la corrección de deformaciones se han elaborado diferentes facsímiles pintados al óleo sobre un soporte de tela de lino Velázquez, con una longitud de 20/50 mm y un ligamento de tafetán simple y trama abierta. Se ha elegido una preparación artesanal de yeso extra de Bolonia y cola de conejo, a la que se ha adicionado una pequeña proporción de pigmento Sombra Tostada, con la finalidad de reproducir la técnica pictórica del caso de estudio real para realizar las pruebas previas a la intervención en ella. Los cien imanes S-0503-N, colocados en el canto exterior del bastidor, permiten sujetar cuatro bandas de tela sintética LIPARI, con un gramaje $\left(\mathrm{g} / \mathrm{m}^{2}\right)$ 260. La tela solapa sobre los bordes del facsímil y se han distribuido 84 imanes Q-20- 
10-02-N en todo el perímetro del conjunto, por ambas caras [figura 5].

El uso diferenciado de los imanes distribuidos perimetralmente a lo largo de la obra, respecto a los imanes empleados en los cantos exteriores es debido a su forma, dimensión y propiedades físicas. Los imanes Q-20-10-02-N son esenciales en esta fase de intervención dada su forma y dimensión, ya que abarca más superficie de contacto con la obra. La distancia y orientación de los imanes es fundamental, ya que con una distancia corta los imanes podrían atraerse creando daños en la obra. Cada imán tiene que estar orientado respecto al contiguo con el polo que se repele; la distancia a respetar entre ellos es la que marca el propio imán por la repulsión que se produce.

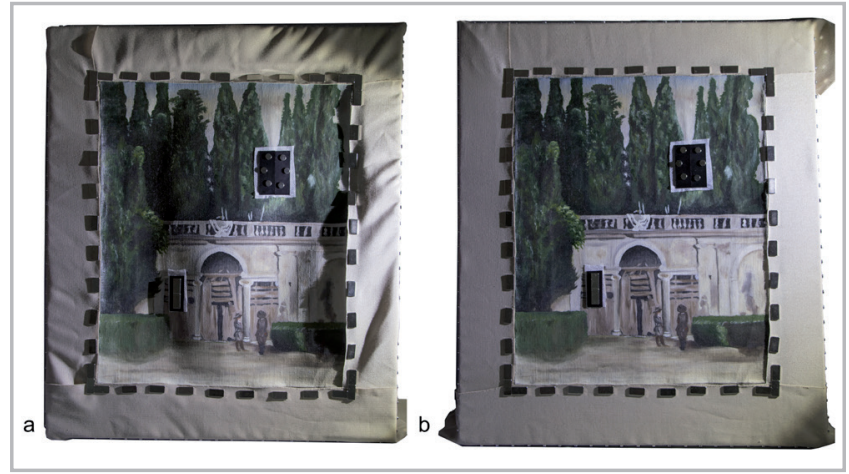

Figura 5.- a) Facsímil pintura al óleo previo al tensado. Luz rasante. b) Facsímil pintura al óleo después de 24 horas de tensión. Luz rasante. @Emanuel Sterp Moga

A continuación, se ha aplicado humedad mediante vapor de ultrasonidos para relajar las fibras y poder efectuar el proceso de tensado. La tensión empleada con el sistema de varillas y tuercas oscila entre 2,5 y $3,5 \mathrm{~N} / \mathrm{cm}$ y se ha mantenido durante 24 horas. Para determinar el rango de tensión se han seguido las indicaciones aportadas por laccarino, quien señala como valor adecuado $1,5 \mathrm{~N} / \mathrm{cm}$ a $2,6 \mathrm{~N} / \mathrm{cm}$, siendo aceptable para algunos casos hasta 3,4 $\mathrm{N} / \mathrm{cm}$ (laccarino 2009). Finalmente, se ha decidido probar la máxima tensión que soportan los imanes Q-20-1002-N durante el proceso de tensado para valorar si la obra estaría en riesgo de sufrir algún daño bajo los niveles máximos. La medición se ha llevado a cabo con el aparato Tensión Meter HT-6510N, obteniendo los datos en N/cm².

Es importante conocer también cómo se debe proceder para separar los imanes y qué fuerza es preciso emplear para lograrlo. La separación se debe realizar deslizando el imán hacia los lados y no hacia arriba, ya que de ese modo la fuerza a emplear es mucho menor. En el caso de los imanes Q-20-10-02-N es preciso ejercer una fuerza de $3,7 \mathrm{~N}$.

-Prueba B. Se ha considerado conveniente contemplar cómo funcionaría el mecanismo basado en el sistema imantado si se tuviese que sujetar temporalmente el lienzo pintado para someterlo a otro tipo de intervenciones como podrían ser suturas hilo a hilo, colocación de puentes de refuerzo o intarsia textil. Para ello, se ha colocado en los cantos interiores del BASM una plancha que quede perfectamente encajada en sus ranuras. Se ha seleccionado una lámina de acero inoxidable, con unas dimensiones de $47 \times 39$ $\times 0,2 \mathrm{~cm}$, que dispone de unos agujeros de $2 \mathrm{~mm}$ de diámetro en toda la superficie para favorecer el campo magnético entre dos imanes y la circulación del aire, una vez depositado el lienzo sobre ella. De nuevo, es importante señalar cómo la fuerza de sujeción puede verse condicionada por la distancia establecida entre los dos imanes que actúan como pinza. De esta manera, se podrá colocar el lienzo pintado sobre la lámina de acero inoxidable, una vez desmontado de su bastidor original, y montarlo provisionalmente con una mínima tensión mediante los imanes de neodimio S-15-03-R [figura 6]. Con este sistema evitaremos grapar o clavetear el orillo perimetral del soporte textil y reduciremos los posibles riesgos durante la manipulación, además de garantizar la reversibilidad y la mínima intervención sobre el original.

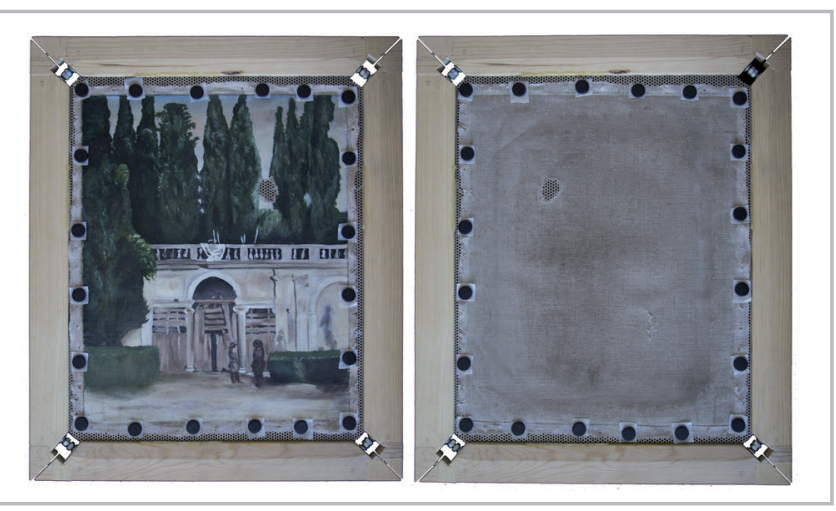

Figura 6.- Bastidor Auxiliar con Sistema Magnético (BASM), sujeción perimetral de la obra mediante imanes de neodimio. (c) Emanuel Sterp Moga

\section{Resultados y discusión}

En relación a las pruebas de tracción del ensayo 1-realizadas para determinar el comportamiento de los adhesivos Pattex ${ }^{\circledast}$ Nural 27 y Araldite ${ }^{\circledR}$ Standard extra fuerte-, cabe señalar que los datos obtenidos con los imanes testados S-12-3-N, S-8-2-N, S-10-2-N, S-5-8-N, S-5-3-N, probados sobre la maqueta $A$, determinaron que se optase finalmente por considerar el adhesivo Araldite ${ }^{\circledR}$ Standard extra fuerte como el más adecuado, debido a que resiste el doble de la fuerza de tracción. En el caso de los imanes S-05-03-N, presentaban una resistencia máxima a la tracción de $28,3 \mathrm{~N}$ mediante el adhesivo Pattex ${ }^{\circledR}$ Nural 27 y de $60 \mathrm{~N}$ con el adhesivo Araldite ${ }^{\circledR}$ Standard extra fuerte. Por otro lado, con los imanes S-12-03-N se obtuvo una resistencia máxima a la tracción de 66,7 N mediante el adhesivo Pattex ${ }^{\circledR}$ Nural 27 y de $139,4 \mathrm{~N}$ en el caso del adhesivo Araldite ${ }^{\circledR}$ Standard extra fuerte. Atendiendo a estos resultados se han elegido los 
imanes S-05-03-N para la construcción del prototipo BASM en maquetas y los imanes S-12-03-N para el prototipo BASM en obra real, debido a que son los imanes que más fuerza de sujeción muestran en relación a su tamaño. Se ha observado que los dos adhesivos testados tienen un fallo de adhesión con el imán cuando estos alcanzan su máxima fuerza de tracción; esto se produce debido a que la resina sintética, al polimerizar, se adhiere con mayor fuerza al soporte leñoso de los listones que componen el bastidor auxiliar que a la base del imán

Respecto a las pruebas efectuadas para medir el comportamiento mecánico de las telas a la tracción mecánica en el ensayo 2, los resultados obtenidos en la maqueta $T$ indican que todas las muestras sometidas a una tensión constante durante $\mathbf{4 8}$ han mantenido su tensión inicial. Se ha considerado más apropiado la utilización de los imanes S-5-3-N para la maqueta del BASM y de los imanes S-12-3-N para el prototipo del BASM, mediante la tela sintética (LIPARI $\mathrm{g} / \mathrm{m}^{2}$ 260) [tabla 1], debido a la relación de tamaño/fuerza que prestan sobre la superficie textil. Con la finalidad de comprobar si las telas pierden tensión durante un tiempo más amplio, se han sometido a una tensión constante durante 7 días. Los resultados dan diferentes valores de entre 0.049 a $0.014 \mathrm{~N}$ y seguramente podrían deberse a una ligera variación en el porcentaje de
$H R$, ya que no parece que los imanes pierdan la fuerza a corto plazo. Se estima que estos cambios son mínimos e insignificantes para una intervención que precise pocas horas de duración.

Por último y en relación con el punto anterior, hay que tener en cuenta la tracción que ejercerán las telas que aguantan los imanes. En el caso de las muestras sujetas con los imanes S-05-03-N se ha observado que soportan una tensión máxima de $3 \mathrm{~N}$, y en las que se han empleado los imanes S-12-03-N, este valor alcanza $12.5 \mathrm{~N}$; a partir de esta fuerza de tensión, los imanes que actúan como pinza dejan de ser eficaces.

Para comprobar si los estratos pictóricos habían experimentado alguna deformación debido a la presión que generan los imanes de tierras raras, se ha observado la superficie de estos con ayuda de un Microscopio digital de alta resolución (Dino-Lite). Los imanes que han sido elegidos para ser colocados sobre las áreas dañadas del soporte textil, por la formación de desgarros o lagunas, han sido S-8-2-N y Q-20-10-02N. Se ha tenido la precaución de proteger la superficie de contacto con una tira de Tyvek ${ }^{\circledR}$ y cinta de neopreno. En la tabla 2 se recogen los datos obtenidos tras las pruebas llevadas a cabo en las maquetas $P$.

Tabla 1.- Fuerza máxima de tracción ejercida en las diferentes telas sujetas con los respectivos imanes. @ Emanuel Sterp Moga

\begin{tabular}{|c|c|c|c|c|c|}
\hline Tipo de telas & Nr. de imancs & $\begin{array}{l}\text { Tipo de } \\
\text { imán }\end{array}$ & $\begin{array}{c}\text { Fucrza } \\
\text { inicial en } \\
\mathrm{Kg}\end{array}$ & $\begin{array}{c}\text { Fuerza } \\
\text { tras } 48 \mathrm{~h} \\
\text { en } \mathrm{Kg}\end{array}$ & $\begin{array}{l}\text { Fucrza tras } \\
7 \text { días en } \mathrm{Kg}\end{array}$ \\
\hline \multirow{3}{*}{$\begin{array}{c}\text { LIPARI } \\
\text { Gramaje }\left(\mathrm{g} / \mathrm{m}^{2}\right) \\
260 \\
\text { Sintética }\end{array}$} & 1 & S-12-3-N & 0.470 & 0.470 & 0.463 \\
\hline & 2 & S-12-3-N & 0.975 & 0.975 & 0.970 \\
\hline & 3 & S-12-3-N & 1.275 & 1.275 & 1.271 \\
\hline \multirow{3}{*}{$\begin{array}{c}\text { ISPRA } \\
\text { Gramaje }\left(\mathrm{g} / \mathrm{m}^{2}\right) \\
130 \\
\text { Sintética }\end{array}$} & 1 & S-12-3-N & 0.580 & 0.580 & 0.577 \\
\hline & 2 & S-12-3-N & 0.670 & 0.670 & 0.665 \\
\hline & 3 & S-12-3-N & 0.760 & 0.760 & 0.751 \\
\hline \multirow{3}{*}{$\begin{array}{c}\text { Lino } 2297 \\
\text { Gramaje }\left(\mathrm{g} / \mathrm{m}^{2}\right) \\
170 \\
\text { Natural }\end{array}$} & 1 & $\mathrm{~S}-12-3-\mathrm{N}$ & 0.440 & 0.440 & 0.432 \\
\hline & 2 & S-12-3-N & 0.665 & 0.665 & 0.658 \\
\hline & 3 & S-12-3-N & 1.235 & 1.235 & 1.225 \\
\hline \multirow{3}{*}{$\begin{array}{c}\text { ISPRA } \\
\text { Gramaje }\left(\mathrm{g} / \mathrm{m}^{2}\right) \\
100 \\
\text { Sintética }\end{array}$} & 1 & S-12-3-N & 0.375 & 0.375 & 0.360 \\
\hline & 2 & S-12-3-N & 0.515 & 0.515 & 0.504 \\
\hline & 3 & S-12-3-N & 0.835 & 0.835 & 0.827 \\
\hline
\end{tabular}

\begin{tabular}{|c|c|c|c|c|c|}
\hline Tipo de telas & Nr. de imanes & $\begin{array}{l}\text { Tipo de } \\
\text { imán }\end{array}$ & $\begin{array}{c}\text { Fuerza } \\
\text { inicial en } \\
\mathrm{Kg}\end{array}$ & $\begin{array}{l}\text { Fuerza } \\
\text { tras } 48 \mathrm{~h} \text { en } \\
\mathrm{Kg}\end{array}$ & $\begin{array}{l}\text { Fuerza tras } \\
7 \text { días en } \mathrm{Kg}\end{array}$ \\
\hline \multirow{3}{*}{$\begin{array}{c}\text { LIPARI } \\
\text { Gramaje }\left(\mathrm{g} / \mathrm{m}^{2}\right) \\
260 \\
\text { Sintética }\end{array}$} & 1 & $S-5-3-N$ & 0.095 & 0.095 & 0.090 \\
\hline & 2 & S-5-3-N & 0.160 & 0.160 & 0.152 \\
\hline & 3 & S-5-3-N & 0.240 & 0.240 & 0.236 \\
\hline \multirow{3}{*}{$\begin{array}{c}\text { ISPRA } \\
\text { Gramaje }\left(\mathrm{g} / \mathrm{m}^{2}\right) \\
130 \\
\text { Sintética }\end{array}$} & 1 & S-5-3-N & 0.090 & 0.090 & 0.084 \\
\hline & 2 & S-5-3-N & 0.175 & 0.175 & 0.169 \\
\hline & 3 & S-5-3-N & 0.285 & 0.285 & 0.280 \\
\hline \multirow{3}{*}{$\begin{array}{c}\text { Lino } 2297 \\
\text { Gramaje }\left(\mathrm{g} / \mathrm{m}^{2}\right) \\
170 \\
\text { Natural }\end{array}$} & 1 & S-5-3-N & 0.090 & 0.090 & 0.082 \\
\hline & 2 & S-5-3-N & 0.155 & 0.155 & 0.142 \\
\hline & 3 & S-5-3-N & 0.265 & 0.265 & 0.256 \\
\hline \multirow{3}{*}{$\begin{array}{c}\text { ISPRA } \\
\text { Gramaje }\left(\mathrm{g} / \mathrm{m}^{2}\right) \\
100 \\
\text { Sintética }\end{array}$} & 1 & S-5-3-N & 0.095 & 0.095 & 0.091 \\
\hline & 2 & S-5-3-N & 0.170 & 0.170 & 0.164 \\
\hline & 3 & S-5-3-N & 0.245 & 0.245 & 0.238 \\
\hline
\end{tabular}

Tabla 2.- Valoración de los daños causados por la presión de los imanes sobre diferentes capas pictóricas. Verde: sin daños; Amarillo: escasos daños; Naranja: algún daño; Rojo: daños graves @ Emanuel Sterp Moga

\begin{tabular}{|c|c|c|c|c|c|c|c|c|c|}
\hline & & $\begin{array}{l}\text { S-15- } \\
\text { 03-R }\end{array}$ & $\begin{array}{c}\text { Q-08- } \\
08- \\
04-\mathrm{N}\end{array}$ & $\begin{array}{c}\text { Q-10- } \\
10- \\
03-N\end{array}$ & $\begin{array}{c}\text { S- } \\
12- \\
03- \\
\text { N }\end{array}$ & $\begin{array}{c}\text { S- } \\
10- \\
02- \\
\mathrm{N}\end{array}$ & $\begin{array}{l}\text { S- } \\
08- \\
02- \\
\text { N }\end{array}$ & $\begin{array}{c}\text { Q-25- } \\
06- \\
02- \\
\text { SN }\end{array}$ & $\begin{array}{c}\text { Q-20- } \\
10- \\
02-\mathrm{N}\end{array}$ \\
\hline $\begin{array}{l}\text { Película pictórica } \\
\text { muy fina de óleo sin } \\
\text { preparación }\end{array}$ & Tyvek ${ }^{\circledR}$ & & & & & & & & \\
\hline $\begin{array}{l}\text { Película pictórica de } \\
\text { óleo y preparación }\end{array}$ & Tyvek® & & & & & & & & \\
\hline $\begin{array}{l}\text { Película pictórica de } \\
\text { óleo y preparación }\end{array}$ & $\begin{array}{l}\text { Tyvek® y cinta } \\
\text { de neopreno }\end{array}$ & & & & & & & & \\
\hline
\end{tabular}


En cuanto a la tensión aplicada sobre los facsímiles durante el proceso de corrección de deformaciones en el lienzo, indicar que esta ha oscilado entre 2,5 y 3,5 N/ $\mathrm{cm}$, considerándose un valor suficiente para lograr los objetivos previamente marcados [figura 5 b].

Los resultados de las pruebas realizadas para conocer la tensión lateral máxima experimentada muestran que, a partir de $6,4 \mathrm{~N} / \mathrm{cm}$, los imanes que sujetan la tela empiezan a ceder y la tela se desplaza. Aunque no es una tensión muy alta que ponga en peligro la obra, se ha considerado que esta debía ser la tensión máxima a aplicar durante el uso de los imanes Q-20-10-2-N con S-5$3-\mathrm{N}$, estimando con ello un rango de seguridad.

La fuerza de atracción entre los imanes es esencial ya que de ello depende la fuerza de tracción que se podrá ejercer durante el proceso de tensado. Si se emplean imanes con una fuerza de atracción inferior a los elegidos, no se lograría un buen resultado, pero si se eligiesen imanes más fuertes que los testados en esta investigación, el riesgo de causar algún daño a la obra es muy elevado, debido a que los imanes proporcionarían una fuerza de sujeción mayor y, por tanto, mayor será la tensión.

Respecto a la sujeción perimetral del lienzo con los imanes de neodimio S-15-03-R tras ensayar el proceso no se aprecia que el facsímil haya sufrido ningún daño. Además, el sistema permite una rápida colocación, mantiene el soporte textil en contacto con la plancha metálica y garantiza una buena seguridad impidiendo movimientos durante la manipulación. La tensión mínima que se precisa para sujetar el facsímil evita que el soporte pueda sufrir algún cambio dimensional como consecuencia de posibles variaciones en las condiciones medio ambientales durante el tiempo que dure la intervención; se evita, además, tener que recurrir a la aplicación de pesos.

\section{Aplicación del BASM a un caso de estudio}

Obtenidos todos los datos derivados de las pruebas experimentales, se ha procedido a llevar a cabo la restauración del lienzo pintado seleccionado. El soporte original es una tela de lino y el paño está cortado en una sola pieza; tiene un ligamento tipo tafetán y sus hilos son gruesos. En general, las fibras textiles muestran un estado debilitado por causa del envejecimiento natural de la celulosa potenciado por las condiciones inadecuadas de exposición a las que ha estado expuesto el cuadro. La sujeción perimetral del lienzo se ha visto seriamente comprometida debido a los procesos de oxidación de los clavos usados durante su montaje y sujeción a los travesaños del bastidor. La rotura de las fibras del tejido en las áreas circundantes a la cabeza del elemento metálico ha provocado la aparición de deformaciones locales.
Para la construcción del BASM ha servido de modelo el

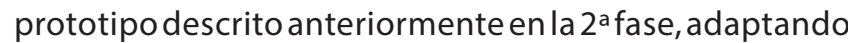
las dimensiones a las necesidades específicas de la obra, $150 \times 110 \times 3 \mathrm{~cm}$. En este caso concreto se han colocado 102 imanes, modelo S-12-03-N, en los cantos exteriores del bastidor auxiliar.

Con la finalidad de corregir las ondulaciones en el tejido, se han empleado 178 imanes, modelo Q-20-10-02- N, que han sido colocados a lo largo de todo el perímetro del cuadro respetando una distancia entre cada imán de $2 \mathrm{~cm}$. Cada pareja de imanes actúa como una pinza para soportar tanto al lienzo original como a las bandas de tela sintética de poliéster LIPARI, con un gramaje $\left(\mathrm{g} / \mathrm{m}^{2}\right) 260$; estas últimas van, a su vez, sujetas por el otro extremo al canto exterior del BASM mediante 102 imanes, modelo S-12-03-N. Para proteger las zonas más vulnerables de la capa pictórica (desgarros, lagunas o rotos) se ha procedido a realizar la metodología desarrollada en las maquetas.

A continuación, se han humectado las fibras del tejido con ayuda de una máquina de vapor de ultrasonidos y tras conseguir la relajación de estas se ha iniciado el proceso de tensado del lienzo. La tensión aplicada al conjunto de la obra oscila entre 2,1 y 3,5 N/cm, durante un periodo de 24 horas. Dicha tensión se ha ido midiendo durante el proceso de corrección de deformaciones [figura $7 \mathrm{c}$ ]. Transcurrido ese intervalo de tiempo se ha observado que las diversas deformaciones iniciales han sido corregidas y el soporte ha recuperado su orografía original [figura $7 \mathrm{~b}$ ].

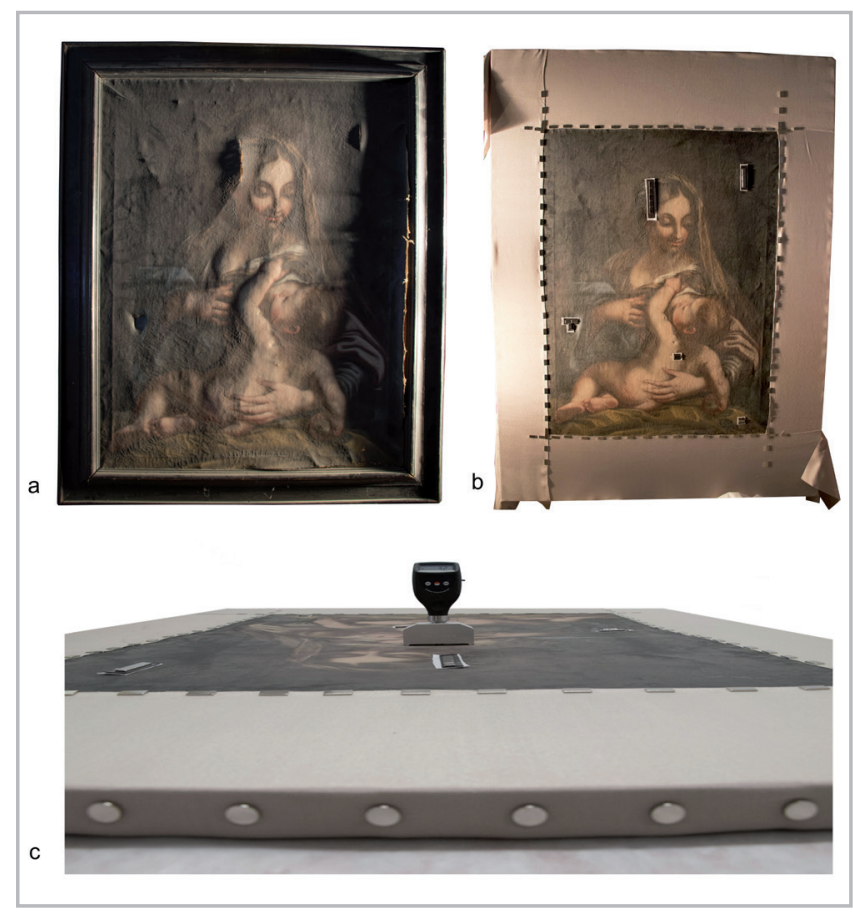

Figura 7- a) Estado inicial del lienzo. Luz rasante; b) Resultado obtenido después de $24 \mathrm{~h}$. de tratamiento. Luz rasante; c) Medición de la tensión durante el proceso de corrección de deformaciones. (c) Emanuel Sterp Moga 
El mecanismo propuesto en este trabajo permite mantener el conjunto del lienzo pintado tensado sobre este dispositivo para efectuar otros tratamientos de restauración que se consideren necesarios [figura 8]. Se aconseja colocar una plancha de acero inoxidable, siguiendo el modelo experimental testado previamente, de modo que aporte una superficie de apoyo. Para proteger la capa pictórica y evitar el contacto directo con la plancha metálica, se ha colocado una lámina de Tyvek ${ }^{\circledR}$. En el caso estudiado, el soporte textil se mantuvo sujeto al bastidor auxiliar bajo una tensión mínima aportada por los imanes durante un total de cuatro semanas, observándose un buen comportamiento del mecanismo y ningún efecto nocivo sobre el cuadro.

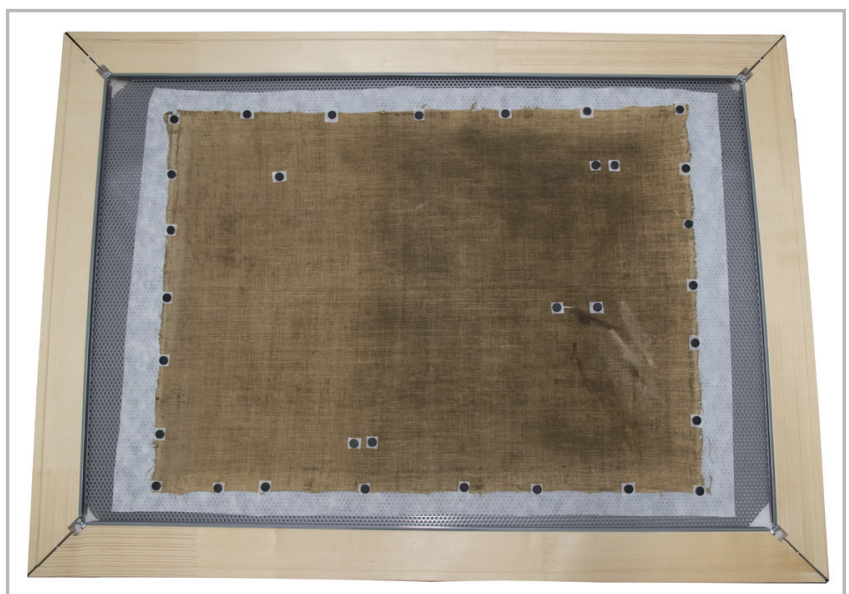

Figura 8-Sujeción perimetral provisional de la obra. @ Emanuel Sterp Moga

\section{Conclusiones}

Los testados llevados a cabo con diferentes telas, adhesivos e imanes y las pruebas realizadas sobre los facsímiles, han permitido conocer el comportamiento de los materiales y valorar cuál era la opción más adecuada para su aplicación en la obra real a restaurar.

El método de montaje del sistema magnético, la fuerza de sujeción y de tracción, el tamaño y el peso de los imanes, son factores que deben ser valorados por el restaurador y ajustados a las características propias de la obra a intervenir.

La elaboración del modelo fotogramétrico y los diferentes estudios virtuales de los modelos desarrollados han sido herramientas de apoyo fundamentales para buscar soluciones a implementar en el proceso de intervención sobre el caso de estudio seleccionado.

La utilización del BASM posibilita minimizar la manipulación del cuadro, evita tener que recurrir a otros métodos más invasivos durante los tratamientos de conservaciónrestauración y garantiza un procedimiento totalmente re-tratable. Por todo lo expuesto, se considera que esta es una herramienta que puede ofrecer al restaurador opciones eficaces y respetuosas con el original.

\section{Bibliografía}

AA.VV. (2005). "El soporte textil: comportamiento, deterioro y criterios de intervención." Actas del Seminario Internacional de conservación de pintura. Valencia: Universitat Politècnica de Valencia.

AA.VV. (2018). Criterios de intervención en pintura de caballete. Proyecto COREMANS. Ministerio de Cultura y Deporte. NIPO: 030-18-136-5.

ACKROYD, P. y BOMFORD, D. (1999). "Questions of reversibility in the conservation of paintings on canvas". En Oddy, A. y Carroll, S. (Ed.) Reversibility - Does It Exist? British Museum Occasional paper number 135. Londres: British Museum, 53-62. ISBN: 0861591356.

APPELBAUM, B. (1987). "Criteria for treatment: Reverssibility". JAIC Journal of the American Institute for Conservation, 26, num.2, Article 1, 65-73.

ART GALERY NEW SOUTH WALES. (2015). A Stella return. https://www.artgallery.nsw.gov.au/blog/posts/a-stella-return/ [Consulta: 26-02-2018]

BESTETTI, R. (2005). "Restauro strutturale e trattamento delle lacune, il caso del dipinto Giardini Romani di Giacomo Balla". Cristina Acidini Luchinat (Ed.). En III Congresso Nazionale IGIIC Lo Stato dell'Arte. Palermo: Nardini, 336-343. ISBN: 8840441441, 9788840441443.

CARITÀ, R. (1955). "Aggiunta sui telai per affreschi trasportati", Bollettino dell'Istituto Centrale del Restauro, 23-24: 165-170.

CHARTERIS, L. (1991). "Reversibility - Myth and Mis-use". En Oddy, A. y Carroll, S. (Ed.) Reversibility - Does It Exist? British Museum Occasional paper number 135. Londres: British Museum, 141145. ISBN: 0861591356.

DERBYSHIRE, A. (2005). "The new miniatures gallery", V\&A Conservation Journal, 51: 2-4. ISSN 9670-2273.

FABEIRO, M. L., HAMADA, S., ILLÁN, A., ROMERO, R. (2005). "Revisión crítica de los diversos tipos de tensión continua aplicados a obras sobre lienzo. Caso práctico realizado en dos obras de Pablo Legot". En II Congreso de GEIIC. Investigación en Conservación y Restauración, Barcelona, Actas en CD, Universidad de Barcelona.

GIRONÉS, I.S., IACCARINO, I.A.,SERINO,C. (2010)."Reflexiones sobre los problemas, las soluciones y los resultados de la manipulación de los grandes lienzos de la Galería Dorada del Paular Ducal de Gandía", Arché Instituto universitario de restauración del patrimonio de la UPV, 4 y 5: 209-210. ISSN: 1887-3960.

HEDLEY, G. A. (1988). "Relative humidity and the stress/strain response of canvas paintings: uniaxial measurements of naturally aged samples". Studies in conservation, 33, 3: 133-148. DOI: 10.1179/sic.1988.33.3.133. 
IACCARINO, I. A. (2009). "About the choice of tension for canvas paintings". Ceroart, Les Dilemmes de la Restauration, 4. http:// journals.openedition.org/ceroart/1269\#entries [Consulta: 28-032018]

MECKLENBURG, M. F. (1982). Some aspects of mechanical behaviour of fabric supported canvas paintings: Report to the Smithson ian Instituion-Research Supported under the National Museum Act, unpublished.

NOEHLES, M., NIEHUS, L. (2014). “Mounting Works on Paper with Neodymium Magnets", Restaurator, International Journal for the Preservation of Library and Archival Material, 35 (3-4): 231-248. ISSN 0034-5806.

RELLA, L. Y SACCANI, L. (2006). "Un restauro ragionato. La Crocifissione di Stephan Kessler". Saonara: II Prato, CESMAR7 Cuaderno N.3. ISBN 88-89566-59-0.

SÁNCHEZ ORTIZ, A. (2012). Restauración de obras de arte: pintura de caballete. Tres Cantos (Madrid): Akal. ISBN 978-84-460-3110-9.

SMITH, R.D. (1988). "Reversibility: a questionable philosophy", Restaurator ,9, 199-207.

SPICER, G. (2010). “Defying Gravity with Magnetism”. AIC News, 35 (6): 1-5. ISSN 1060-3247.

SPICER, G. (2017). "The principles of creating a magnetic mounting system: the physics every conservator needs to know". In Conference: ICON Textile Group Forum. From Boxes to Buildings: Creative Solutions for the Storage of Textiles and Dress, At Bath, UK. https://www.researchgate.net/publication/321170997 The principles of creating a magnetic mounting system the physics every conservator needs to know [Consulta: 28-042018]

STERP, E. (2018). Mínima intervención en pintura sobre lienzo. Incorporación de imanes de neodimio en procesos de conservaciónrestauración. (Trabajo Fin de Máster). Máster en Conservación del Patrimonio Cultural. Universidad Complutense de Madrid, España.

TIPLER, P. A. (1992). Física: preuniversitaria. Tomo II. Barcelona: Reverté, S.A. ISBN: 84-291-4376-9.

TSANG, J., MADRUGA, I. C. C., WILLIAMS, D. PELASARA, R., PATTERSON, R. (2013). "Modernized Stretcher for Paintings on Canovas: Assessment and Observation", AIC Paintings Specialty Group Postprints, 26: 92-94. ISSN: 2372-1634.

VILLERS, C. (ed.) (2003). Lining Paintings: Papers from the Greenwich Conference on Comparative Lining Techniques. London: Archetype Publications. ISBN: 1873132042.

WROCZYNSKI, E. (2013). Conserving a Giant: Resurrecting Pietro da Cortona's Triumph of David. https:// emilywroczynskiconservationportfolio.format.com/gallery\#39 [Consulta: 26-02-2018]

\section{Autor/es}

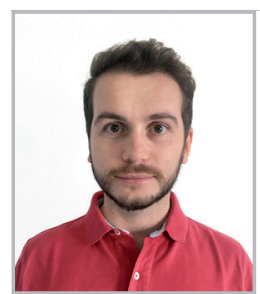

\section{Emanuel Sterp Moga}

emasterp@ucm.es

Doctorando, Facultad de Bellas Artes Universidad Complutense de Madrid.

Emanuel Sterp Moga es Graduado en Conservación y Restauración del Patrimonio Cultural por la Universidad Complutense de Madrid. Obtuvo el galardón al Trabajo Final de Grado realizado durante el año 2017 con la Cátedra Autric Tamayo. Miembro de varios proyectos Innova-Docencia en la Universidad Complutense de Madrid. Sus líneas de investigación se centran en la utilización de sistemas magnéticos en pinturas de caballete y la aplicación de tecnologías 3D para la conservación del Patrimonio Cultural, difundiendo los resultados en varios congresos nacionales e internacionales. Actualmente, es doctorando y colaborador honorifico en el del Departamento de Pintura y ConservaciónRestauración de la Universidad Complutense de Madrid.

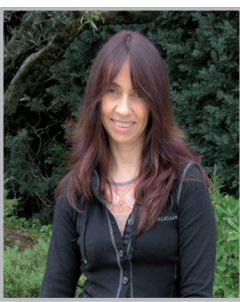

Alicia Sánchez Ortiz
alicisan@ucm.es
Directora del Departamento de Pintura y
Conservación-Restauración de la Facultad
de Bellas Artes. Universidad Complutense
de Madrid.

Alicia Sánchez Ortiz es Doctora en Bellas Artes por la Universidad Complutense. Desde 1993 hasta la actualidad imparte docencia en las diferentes titulaciones relacionadas con la conservación del Patrimonio Cultural que se ofertan en la Facultad de Bellas Artes [UCM]. Directora de varios cursos de Formación Continua y de numerosos Convenios de Investigación Art-83 L.O.U. Investigadora Principal de Proyectos I+D+i competitivos en el ámbito de la conservación del patrimonio científico universitario y de las colecciones de ceroplástica anatómica: HAR2009-10679 y HAR2013-42460-P. Conferenciante y ponente en congresos nacionales e internacionales, ha escrito libros, capítulos, catálogos y artículos de investigación relacionados con la conservación del patrimonio cultural. 